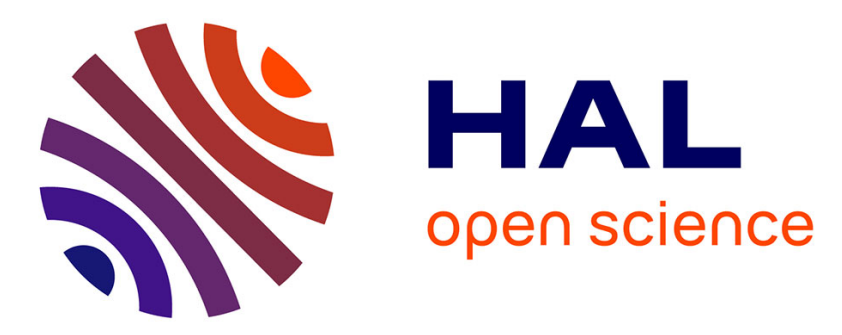

\title{
Optical penetration depth determination in a graphite-epoxy laminate by photoacoustic F.T.I.R. spectroscopy
}

M. Dubois, F. Enguehard, L. Bertrand, M. Choquet, J.-P. Monchalin

\section{- To cite this version:}

M. Dubois, F. Enguehard, L. Bertrand, M. Choquet, J.-P. Monchalin. Optical penetration depth determination in a graphite-epoxy laminate by photoacoustic F.T.I.R. spectroscopy. Journal de Physique IV Proceedings, 1994, 04 (C7), pp.C7-377-C7-380. 10.1051/jp4:1994788 • jpa-00253319

\section{HAL Id: jpa-00253319 https://hal.science/jpa-00253319}

Submitted on 1 Jan 1994

HAL is a multi-disciplinary open access archive for the deposit and dissemination of scientific research documents, whether they are published or not. The documents may come from teaching and research institutions in France or abroad, or from public or private research centers.
L'archive ouverte pluridisciplinaire HAL, est destinée au dépôt et à la diffusion de documents scientifiques de niveau recherche, publiés ou non, émanant des établissements d'enseignement et de recherche français ou étrangers, des laboratoires publics ou privés. 


\title{
Optical penetration depth determination in a graphite-epoxy laminate by photoacoustic F.T.I.R. spectroscopy
}

\author{
M. Dubois, F. Enguehard, L. Bertrand, M. Choquet* and J.-P. Monchalin*
}

Ecole Polytechnique de Montréal, Département de Génie Physique, C.P. 6079, Succ. Centre-Ville, Montréal, Québec, Canada, H3C $3 A 7$

* Institut des Matériaux Industriels, Conseil National de Recherches du Canada, 75 boulevard Mortagne, Boucherville, Québec, Canada, J4B 6Y4

\begin{abstract}
No quantitative spectrum of optical penetration in graphite-epoxy has been so far reported. Transmission and reflection techniques are inefficient or do not give absolute values. We used three methods to compute the absolute optical penetration spectrum from the photoacoustic signal obtained from a graphite-epoxy composite sample. The spectra obtained in the infrared band of 2.5 to $25 \mu \mathrm{m}$ are presented. The experimental results are also compared to those of a theoretical model.
\end{abstract}

\section{INTRODUCTION}

Laser ultrasonics, based on laser generation and detection of ultrasound, is a promising technique for the non-destructive evaluation of materials, especially in the case of composite materials $[1,2]$. Among the physical parameters, the optical penetration depth of the excitation wavelength plays a dominant role in determining the efficiency of conversion of optical energy to acoustic energy as well as the types of ultrasonic waves generated (shear or longitudinal). Thus, it is very interesting to know the absolute optical absorption spectrum of the composite material.

In this paper, we present results of the Fourier Transform InfraRed PhotoAcoustic Spectroscopy (FTIR-PAS) techniques applied to graphite-epoxy laminates. This composite material being opaque in the near-infrared band, the classical transmission technique cannot be applied. A previous work was published presenting the optical penetration depth spectrum obtained by transmission on the epoxy alone [2]. Other techniques based on reflection are hard to exploit quantitatively. Thus, FTIR-PAS seems to be an interesting technique that can adequately provide absolute values of the optical absorption spectra of composites. Three optical penetration spectra, derived from the same experimental signal using three different data processing methods, are presented and compared. We also present an analytical model of the photoacoustic signal for a two layer system.

\section{MATHEMATICAL EXPRESSION OF THE PHOTOACOUSTIC SIGNAL}

McDonald [3] found an expression of the photoacoustic signal which takes the thermal expansion into account. In the case of our composite samples, we found that the thermal expansion contribution to the signal is negligible. We can thus write the expression of the photoacoustic signal in the following manner :

$$
P_{\omega}(t)=K\left(\frac{\beta \mu}{(\beta \mu+1)^{2}+1}+j \frac{(\beta \mu+1) \beta \mu}{(\beta \mu+1)^{2}+1}\right) e^{j \omega t} \quad \mu=\sqrt{\frac{2 \kappa}{\omega}}
$$

where $P_{\omega}(t)$ is component of the photoacoustic signal modulated at $\omega, K$ an experimental constant, $\beta$ the optical absorption coefficient, $\omega$ the angular frequency of the light modulation, $t$ the time and $\mu$ the thermal diffusion length in the sample ( $\kappa$ being its thermal diffusivity).

\section{REAL PART SATURATION METHOD}

This method was first proposed by Choquet et al. [4]. After complex Fourier transformation of the experimental pressure signal, one obtains the real and imaginary parts of the photoacoustic spectrum which must be identified to the theoretical expressions of (1). The study of the shapes of these two 
functions of $\beta \mu$ shows that the real part passes through a maximum for $\beta \mu=\sqrt{2}$, while the imaginary part is a growth function of $\beta \mu$. Thus, if a point is found in the experimental photoacoustic spectrum where the real part passes through a maximum while the imaginary part does not change its behavior, then one can say that at that point $\beta \mu=\sqrt{2}$. It is then easy to calculate the experimental constant $K$ at that point using the expression of either the theoretical real part or the theoretical imaginary part. With this value of $\mathrm{K}$, the $\beta \mu$-spectrum can be calculated. If one knows the thermal diffusion length spectrum, or $\mu$-spectrum, of the sample, one can then derive its optical absorption spectrum, or $\beta$-spectrum.

\section{TWO MIRROR VELOCITY COMPARISON METHOD}

This method, first proposed by Teng et al.[5], exploits only the amplitude $q$ of the photoacoustic signal (1). If one assumes that the value of the velocity of the interferometer moving mirror $v$ only affects those of the thermal diffusion lengths via the modulation frequencies, collecting two photoacoustic signals of the same sample with two different velocities $v_{1}$ and $v_{2}$ will yield two experimental photoacoustic amplitude spectra $q_{1}$ and $q_{2}$. In the expression of the ratio of these two spectra, the experimental constant $\mathrm{K}$ disappears. It is then easy to invert numerically the experimental $\mathrm{q}_{1} / \mathrm{q}_{2}$-spectrum to obtain a $\beta \mu_{1}$-spectrum.

\section{TRANSMISSION CALIBRATION METHOD}

If it is possible to find a reference sample which is transparent enough for a given wavelength, its optical absorption coefficient $\beta$ at that wavelength can be measured by the classical transmission technique. $\beta$ being known, the value of $\beta \mu$ can be determined at that wavelength, and hence one can calculate the experimental constant $K$ of the photoacoustic spectrum of the reference sample. This value of $\mathrm{K}$ is then used to obtain the $\beta \mu$-spectrum of the sample to study from its photoacoustic signal recorded under the same experimental conditions as those for the reference sample.

\section{EXPERIMENTAL CONDITIONS AND RESULTS}
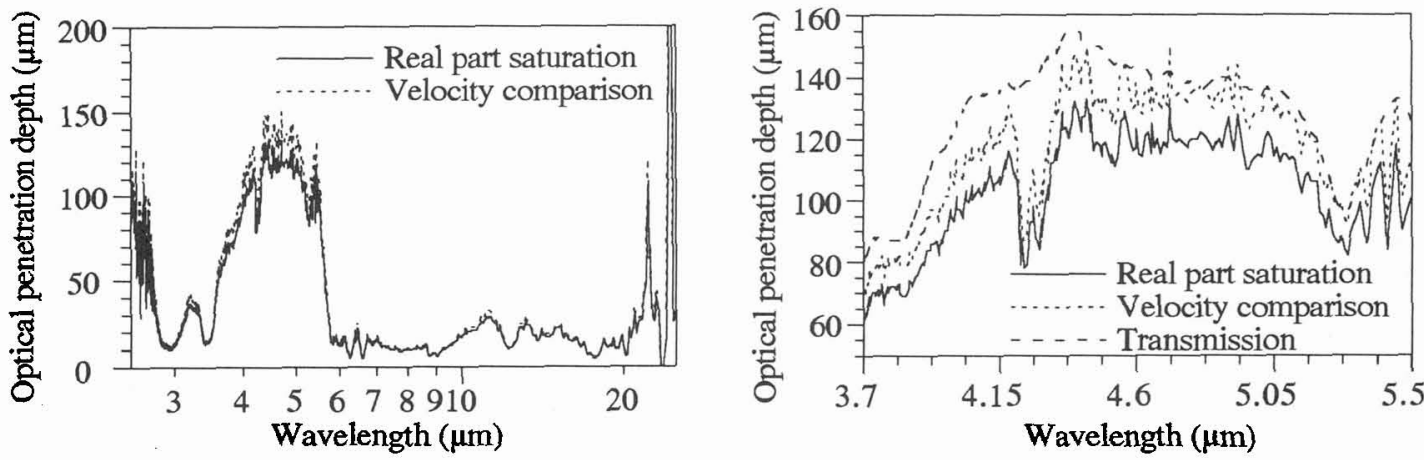

Figure 1 Results of the real part saturation and two mirror velocity comparison methods for the cured epoxy sample.

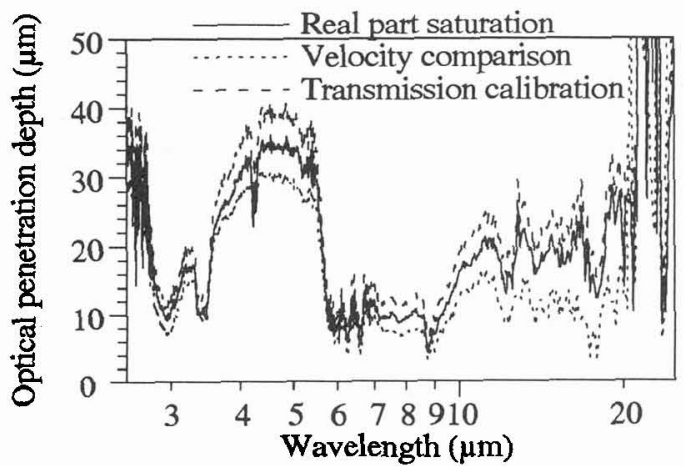

Figure 3 Comparison of the results of the three methods for the graphite-epoxy composite laminate.

Figure 2 Comparison of the photoacoustic methods with the transmission method over the band from 3.7 to $5.5 \mu \mathrm{m}$ for the cured epoxy sample.

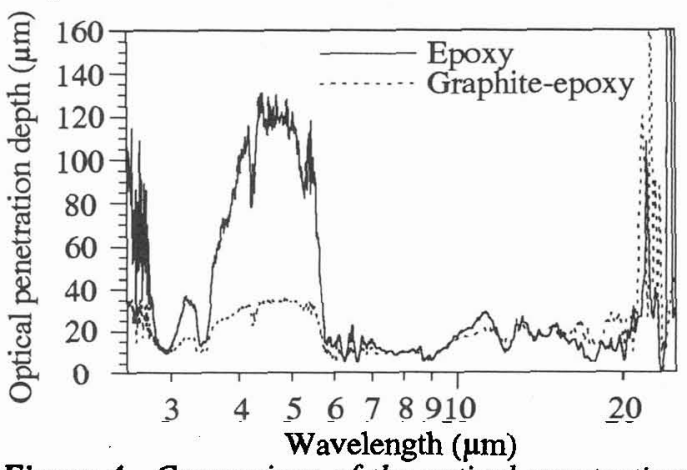

Figure 4 Comparison of the optical penetration depth spectra of the composite laminate and of epoxy. 
The measurements were performed on a graphite-epoxy laminate and a piece of pure cured epoxy. Both samples were provided by the same manufacturer and their epoxies were assumed to be chemically identical. The samples were ground in order to fit the photoacoustic cell. Observations through a microscope did not reveal any modifications of their structures and showed the presence of an epoxy layer approximately $30 \mu \mathrm{m}$ thick covering the top graphite fiber sheet of the composite.

All the experiments were performed with the same apparatus, a BOMEM DA-3 FTIR spectrometer, equipped with a home-made photoacoustic cell. The gas in the cell was helium and the light source was a globar.

Figure 1 presents two optical penetration depth spectra for the epoxy sample. The first one was obtained with the real part saturation method and the second one with the two mirror velocity comparison method.

Figure 2 compares the photoacoustic spectra of the pure epoxy to the transmission technique result over the spectral band from 3.7 to $5.5 \mu \mathrm{m}$. This spectral band is the only interval where the transmission technique can easily be applied on the epoxy sample.

Figure 3 compares the results obtained with the three presented methods on the graphite-epoxy sample. The transmission calibration method was applied using the results obtained on epoxy. epoxy.

Figure 4 compares the optical penetration depth spectrum of the composite laminate to the one of the

\section{SIMULATION OF THE PHOTOACOUSTIC SIGNAL FOR 2 MEDIA ON A BACKING}

In order to check that our composite sample behaves as a simple two-layer system, the first layer being made of epoxy, and the second one being the bulk composite, we developed an analytical 1-D model of the photoacoustic signal for a two-layer system based on the same thermal diffusion equations and boundary conditions as those of Rosencwaig and Gersho [6]. The model considers a sample made of two layers with different thermal and optical properties. We define the following parameters: $I_{0}$ light intensity, $a_{i}=1 / \mu_{i}$ coefficient of thermal diffusion, $k_{i}$ thermal conductivity and $\sigma_{i}=a_{i}(1+j)$ complex coefficient of thermal diffusion. The index i may take the values $1,2,3$ or $g$ indicating the properties of the gas ( $(\mathrm{g})$, of the sample( 1 and 2 ) or of the backing (3). Solving this thermal conduction problem, we obtained the expression $\Psi$ for the amplitude of the gas temperature modulation at the interface medium 1/medium g:

$$
\Psi=I_{0}\left(\frac{k_{1} \beta_{1}\left(\beta_{1}-\sigma_{1}\right)}{\Delta_{1 g}^{-} \gamma_{1}}-\frac{2 k_{1} \sigma_{1}\left(k_{1}\left(\phi_{1}-\phi_{2}-\frac{\beta_{1} \sigma_{1} \Theta}{\gamma_{1} \Delta_{1 g}^{-}}\right) \varepsilon^{+}+k_{2} \sigma_{2}\left(\frac{\beta_{1} \Theta}{\gamma_{1} \Delta_{1 g}^{-}}+\frac{\phi_{2}}{\beta_{2}}-\frac{\phi_{1}}{\beta_{1}}\right) \varepsilon^{-}\right)}{\left(v^{+}+\Phi^{+}\right) \Delta_{23}^{-}+\mathrm{e}^{21_{2} \sigma_{2}}\left(v^{-}+\Phi^{-}\right) \Delta_{23}^{+}}\right)
$$

$$
\text { where: } \begin{aligned}
\Delta_{\mathrm{ij}}^{ \pm}=\left(\mathrm{k}_{\mathrm{i}} \sigma_{\mathrm{i}} \pm \mathrm{k}_{\mathrm{j}} \sigma_{\mathrm{j}}\right) \quad \gamma_{\mathrm{i}}=2 \mathrm{k}_{\mathrm{i}}\left(\beta_{\mathrm{i}}^{2}-\sigma_{\mathrm{i}}^{2}\right) \quad \phi_{\mathrm{i}}=\frac{\beta_{\mathrm{i}}^{2} \mathrm{e}^{1_{1}\left(\sigma_{1}-\beta_{1}\right)}}{\gamma_{\mathrm{i}}} \quad \Theta=\beta_{\mathrm{l}} \mathrm{k}_{\mathrm{l}}-\mathrm{k}_{\mathrm{g}} \sigma_{\mathrm{g}} \\
v^{ \pm}=\left(1-\mathrm{e}^{2 \mathrm{l}_{\mathrm{l}} \sigma_{\mathrm{i}}}\right)\left(\mathrm{k}_{1}^{2} \sigma_{1}^{2} \pm \mathrm{k}_{2} \sigma_{2} \mathrm{k}_{\mathrm{g}} \sigma_{\mathrm{g}}\right) \quad \varepsilon^{ \pm}=\mathrm{e}^{21_{2} \sigma_{2}} \Delta_{23}^{ \pm} \pm \Delta_{23}^{-} \quad \Phi^{ \pm}=\left(1+\mathrm{e}^{21_{\mathrm{l}} \sigma_{\mathrm{l}}}\right) \mathrm{k}_{\mathrm{l}} \sigma_{\mathrm{l}} \Delta_{\mathrm{g} 2}^{ \pm}
\end{aligned}
$$

We present the experimental and calculated ratios of the amplitudes of the photoacoustic signals obtained from the composite over the ones obtained from the pure epoxy. For the calculated signal from the composite, the properties of media $g, 1,2$ and 3 were taken to be respectively those of helium, epoxy, the composite and stainless steel (constituent of the photoacoustic cell). For the calculated signal from the pure epoxy, we gave the properties of epoxy to medium 2. Figure 5 presents the calculated and experimental ratios.

The thermal conductivity of the bulk composite was taken lower than the radial one of the graphite fibers that is suggested in the literature. Due to the existence of the interfacial thermal resistance between the fibers and the matrix, this approach seems valid. The value was taken to be $0.05 \mathrm{~W} / \mathrm{m}^{\circ} \mathrm{K}$.

\section{DISCUSSION}

Concerning the epoxy sample, the results of the two photoacoustic methods are in fairly good agreement with those of the transmission technique, as illustrated by figure 2 . The main difference is the presence of a doublet around $4.24 \mu \mathrm{m}$ in the photoacoustic spectra. This doublet is attributed to the antisymmetric stretching of the $\mathrm{C}=\mathrm{O}$ binding in $\mathrm{CO}_{2}$ [7], which could be present in the photoacoustic cell as a contaminant of the helium. Outside this band, the agreement between the spectra obtained with the two photoacoustic methods is good (figure 1). Moreover, these spectra agree with those obtained using other techniques [8] on the same kind of sample.

Concerning the composite laminate, figure 3 shows that the real part saturation method and the transmission calibration method give results that are quite compatible. On the other hand, the two mirror 
velocity comparison method gives slightly lower penetration depths, while for the epoxy sample there was no such difference. The two mirror velocity comparison method is based on the assumption that a change in the mirror velocity results only in a change in the thermal diffusion lengths via the modulation frequencies. This assumption may not be valid for a composite material, which may explain the discrepancy between the 2 photoacoustic spectra.

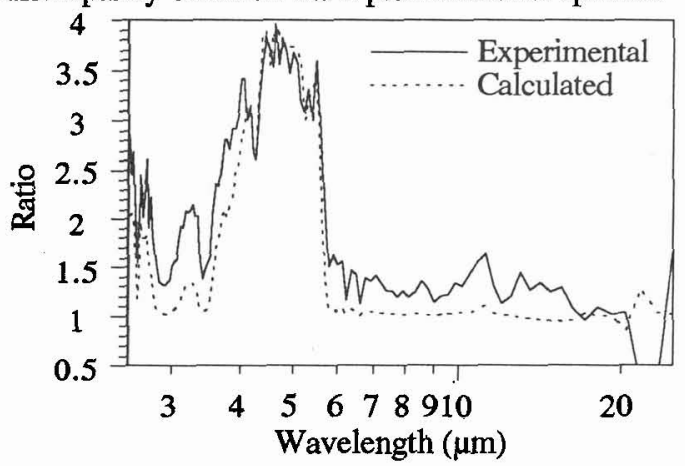

Figure 5 Experimental and calculated ratios of the graphite-epoxy laminate photoacoustic signal over the pure cured epoxy one.

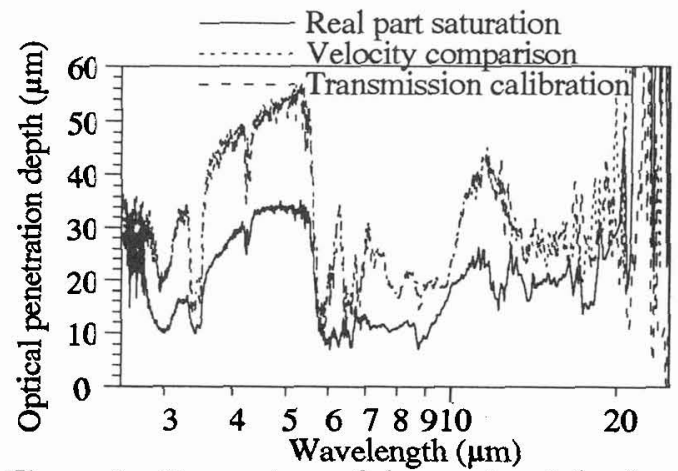

Figure 6 Comparison of the results of the three methods for the painted graphite-epoxy composite laminate.

It can be seen on figure 4 that the optical penetration depths in the spectral band between 3.7 and $5.5 \mu \mathrm{m}$ are of the order of $30 \mu \mathrm{m}$ for the composite instead of $140 \mu \mathrm{m}$ for the epoxy. The optical penetration depth in the composite laminate is approximately equal to the thickness of the epoxy layer above the top fiber sheet. We can thus conclude that, for these wavelengths, the light goes through the epoxy layer and then reaches the first fiber sheet where it is completely absorbed. This interpretation is confirmed by figure 5 , which proves that the composite behaves as a two-layer system, the second layer being highly absorbing.

We made the same experiments on a similar but painted composite. The results are presented on figure 6 . This figure shows that the two mirror velocity comparison and transmission calibration methods give spectra with higher optical penetration depth values than those given by the real part saturation method. The values of the spectra of the two former methods are higher than those obtained with the same methods on the bare composite. The real part saturation of the photoacoustic signal is thus weaker for the painted composite, which makes the identification of the saturation points more error prone.

\section{CONCLUSION}

We have presented three methods to derive the optical penetration depth spectrum from the photoacoustic signal. These three methods allow us to conclude with a good degree of accuracy on the absolute values of the optical penetration depths over the spectral band from 2.5 to $25 \mu \mathrm{m}$.

Photoacoustic spectroscopy seems to be the adequate technique for the absolute determination of the optical absorption spectra of opaque materials like the graphite-epoxy laminate. The knowledge of these spectra will find a direct application in the field of laser generation of ultrasound.

\section{ACKNOWLEDGMENTS}

We wish to thank K.I. McRae, W.R. Sturrock and E. Jensen of the Defense Research Establishment Pacific, National Defense of Canada for supplying the cured epoxy and graphite-epoxy laminate samples.

\section{REFERENCES}

[1] C. Padioleau, P. Bouchard, R. Héon, J.P. Monchalin, F.H. Chang, T.E. Drake, K.I. McRae, Review of Progress in Quantitative NDE, 12 (1993) 1345-1352.

[2] A.D.W. McKie, R.C. Addison Jr., Review of Progress in Quantitative NDE, 12 (1993) 507-516.

[3] F.A. McDonald, J. Opt. Soc. Am., 70, no.5 (1980) 555-557.

[4] M. Choquet, G. Rousset and L. Bertrand, Can. J. Phys., 64, no.9 (1986) 1081-1085.

[5] Y.C. Teng and B.S.H. Royce, Appl. Opti., 21, no.1 (1982) 77-80.

[6] A. Rosencwaig, Photoacoustics and Photoacoustic Spectroscopy, (Krieger Publishing Company, U.S.A. 1990).

[7] G. Herzberg, Molecular spectra and molecular structure, (Van Nostrand, U.S.A., 1945).

[8] K.C. Cole, A. Pilon, D. Noel, J.-J. Hechler, A. Chouliotis, K. C. Overbury, Appl. Spectrosc., 42, no.5 (1988), 761-769. 